\title{
Teaching Smart Materials to Engineering Undergraduate Students: A Problem Solving Approach
}

\author{
Mohammad H. Elahinia \\ Mechanical Engineering Department \\ Virginia Polytechnic Institute and State University \\ Blacksburg, VA 24061
}

\section{Summary and Introduction}

This paper describes a problem solving approach for teaching the subject of smart materials to Mechanical Engineering undergraduate students. An experiment with a Shape Memory Alloy (SMA) actuated robotic arm is designed for the senior undergraduate laboratory (ME4006) in the Department of Mechanical Engineering at Virginia Tech. ME4006 is designed to provide the students with experience in experimental investigation of mechanical engineering systems. In designing the SMAactuated robot experiment it was intended for the students to have a hands-on experiment with Shape Memory Alloys. Furthermore, students learn about sensing and actuation advantages of the SMAs along with their control problems and limitations. The problem is described in a memorandum to the students from a supervisor, who defines the purpose of the problem and defines the audience for the report. Students are not given a procedure to follow for conducting the experiment. In the lab, they design the experiment procedure based on their engineering judgments. Students are introduced to this problem by two lectures which are followed by two sessions of lab time. After performing the experiment the students, working in teams of five, present their results to an audience of faculty, graduate teaching assistants, and two other student teams.

In the case of the SMA-actuated robot, the lecture covers some background on SMAs as well as introducing different applications of these materials. In the memorandum the system is introduced as a prototype robot that will be installed on a mobile platform. The purpose of the mobile robot is to deploy sensors in a hostile environment. Students, as employees of the company, are asked to test the SMAactuated robot for two main goals. The first goal is to choose appropriate PID controller gains such that the robot meets certain criteria in deploying the sensors. The choice of controller gains is also limited by the energy available to the system. The second goal is to investigate the possibility of using the SMA wire as a sensor. Currently, the robot uses an encoder for position feedback. The intent of this second objective is to determine if a relationship between the resistance of the wire and the position of the robot can be used to replace the encoder. The purpose of this paper is to show the effectiveness of a problem solving approach in teaching smart materials to undergraduate engineering student. Lectures, laboratory experiments, and presentations are three essential parts of this approach; together they greatly enhance the learning process. 


\section{Background on ME4006}

As smart materials are changing the practice of Engineering, providing undergraduate engineering students with experiences with these materials has become necessary. To address the educational needs, several engineering departments have developed elective courses or laboratory experiments on smart materials. California State University at Fullerton, for example, has recently established an Intelligent Systems Laboratory to provide Mechanical Engineering students with hands-on experience on integrated design and manufacturing of intelligent systems [1]. As another example, a case study has been done in the Department of Aerospace Engineering at Texas A\&M University. In their study, they have integrated shape memory alloy into a first-year engineering course and a first-year engineering project. The results, that have been reported both qualitatively and quantitatively, showed the successful impact of the experience [2].

ME4006 (Experimental Laboratory II) is a successful, large-enrollment laboratory course in mechanical engineering at Virginia Tech. This course interweaves instruction on engineering principles with instruction on engineering communication [3]. The student presentations are effectively incorporated into the laboratory course. Each presentation follows by students addressing the critiques from the faculty, graduate teaching assistants, and another group of students. Incorporation of the presentations has enhanced the understanding by the students of the technical issues of the laboratory. The course consists of four laboratory problems that students work on in teams, but communicate in individual reports (and for two problems, in team presentations). Each of these problems is described in a memorandum from a supervisor, who defines the purpose of the problem and defines the audience for the communication. For each laboratory problem, students attend two lectures that discuss the engineering principles of those problems and attend two laboratory sessions in which they make measurements to understand and address the problem [4].

What are smart materials? What makes them different from other materials? How can they be used to solve engineering problems and what are the difficulties of their application? Answering these and other similar questions is the focus of the courses on smart materials that are normally offered for graduate students. On the other hand, smart materials are becoming more popular for variety of applications. For example, SMAs are attractive because of their light weight, sensing capability, and being able to provide large actuation force/torque. This brings up the need for Mechanical Engineers to be familiar with these materials and to know the benefits as well as the difficulties of using them. To this end, we found the methodology developed in ME4006 a suitable platform for teaching several aspects of Shape Memory Alloys as an important sub-class of smart materials. The major benefit of using this course as compared to a regular lecture-type course is that the students' learning is considerably enhanced. The enhancement can be attributed to the facts that the students have to design the procedure for running the test and collecting data, they need to look at the problem from multiple perspectives, and as engineers they deal with solving practical issues related to the problem. The other factor that plays an important role in improving the learning is that the students have to present 
their results; these presentations are critiqued by faculty, graduate teaching assistants, and peer students. In the process of preparing the presentations, the behavior of the Shape Memory Alloys as well as other technical aspects of the lab are better understood.

\section{Shape Memory Alloy Experiment}

For this experiment as for the other experiments of the course [4], rather than giving the students a recipe of steps to follow, we give the students the problem, two technical lectures on different aspects of the problem, and two two-hour laboratory sessions to solve the problem. On hand during those laboratory sessions is a graduate teaching assistant who provides advice and makes sure that neither the students nor the equipment is damaged. The experiment setup consists of a Shape Memory Alloy actuated robot instrumented with an encoder for measuring the angular position of the robot. The signal conditioning and power regulation hardware, and the data collection and control design software (MATLAB/dSPACE) are also part of the experiment's setup. The robot, shown in Figure 1, is actuated by a Shape Memory Alloy wire that upon heating undergoes phase transformation and therefore contracts and rotates the moving arm upward. The temperature of the wire changes as the result of the energy balance between the input energy in the form of Joule heating and dissipative energy in the form of natural convection. The SMA wire's contraction is a function of temperature and stress of the material. The position control of the robotic arm is a complicated problem partly due to the fact that the phase transformation takes place between two stressdependent temperatures.

Students attend the first lecture before the first lab session. This lecture is on the background of SMAs, their applications and a review on Proportional-Integral-Derivative (PID) controllers. In the lab the students are to investigate the robot to find a set of gains that enables the PID controller to accurately position the robot while satisfying the energy requirements. Furthermore, the students are to investigate the possibility of using the SMA wire as a displacement sensor. Details of the requirements for the experiment are shown in the problem memorandum presented in Appendix A. Students, acting as the employees of the robotics company, spend the second lecture asking any questions they might have from the company's consultant (the instructor). They return to the lab for the second time to finish collecting data or to repeat some parts of the experiment.

The experiment is designed to cover several engineering disciplines including controls, heat transfer, circuit analysis, signal processing, and the thermomechanical behavior of the Shape Memory Alloys in order to prepare the students for dealing with multi-disciplinary engineering problems with their solution mostly being the compromise between several design-restrains. Also, the memorandum is prepared in a way that the problem does not have unique, straight-forward answers. For example, the restriction on the amount of electrical energy that the robot can use, over the period of one hour, to perform its task makes the search for control gains an iterative process. Instead of going 
through this rather time consuming process some students learned to design a rapid test procedure in order to check whether or not all the design requirements can be satisfied. They measured the amount of electrical power that the SMA-actuated robot consumes to steadily hold the pay-load and compared it with the energy consumption limit. Performing this test showed the need to compromise between the task and energy requirements.

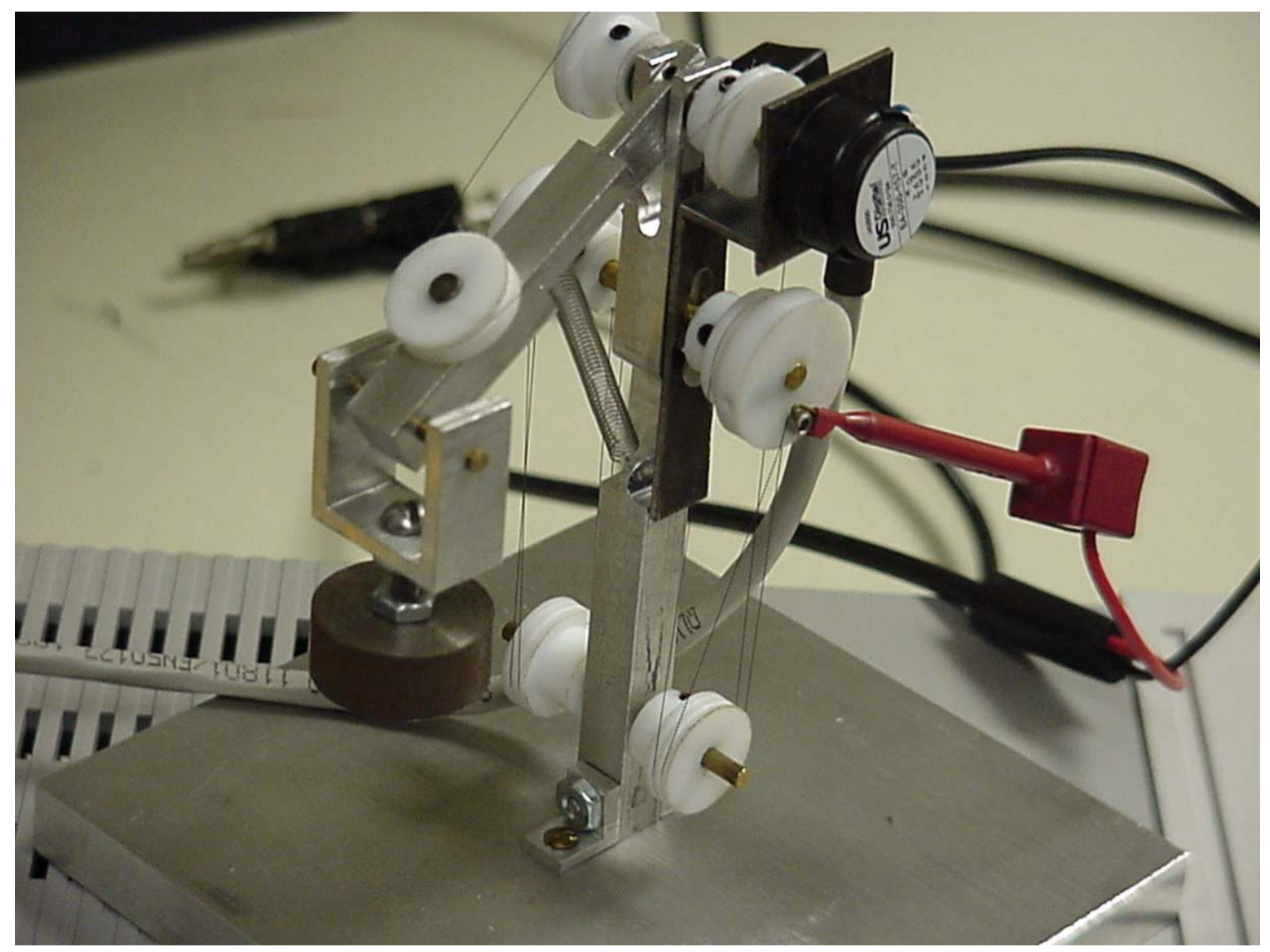

Figure 1 Laboratory set-up for the Shape Memory Alloy experiment. For this problem, students must measure the voltage and current of the SMA wire to find the proper gains for a position controller.

The control of SMA actuators, due to their nonlinear behavior, is a complex problem and the subject of ongoing research [5]. Designing a PID control system for an SMA-actuated system is primarily a hands-on control experience for the students. The lab's setup allows the students to investigate the effect of each set of control gains on the closed-loop response of the robot. Performing the experiment also gives the students the opportunity to see the shortcomings of linear controllers in controlling nonlinear systems. Due to the nonlinearity of the system (the robot) and the actuator (SMA wire), the PID controller cannot provide uniform performance. In other words the same set of gains cannot position the robot in all desired angular positions. Nonetheless, it is possible to have a nonlinear controller made of multiple sets of PID gains and a scheme for switching between these gains. This type of controller, which was suggested by a group 
of students who were unable to obtain acceptable performance with PID controller, is an effective control design.

Several properties of the SMAs change as they undergo phase transformation. To make this fact more accessible to the students we added a part to the experiment in which the change in the resistance SMA wire, as the result of phase transformation, is used as a replacement for the angular position sensor. To this end, the students characterize the hysteresis of the SMA wire while it actuates the robot. In the process of making decisions to replace the encoder with a relationship between the resistance of the SMA wire and the angular position of the robot, the students need to correlate the characteristic of the hysteresis with the closed-loop response restrictions.

\section{Results of the Problem Solving Approach}

The ME undergraduate students do not have a course on smart materials. This experiment provides an opportunity for them to become familiar with smart materials, in general, and Shape Memory Alloys, in particular, without having a one-semester-long course that would cover details. Using a problem solving approach, in the form of a laboratory experiment, for teaching smart materials to the undergraduate student has several advantages including:

1. The experiment presents the students with a real-life engineering problem. In solving the problem they need to utilize multiple disciplines including controls, heat transfer, circuit design, and Shape Memory Alloy thermomechanical behavior.

2. As is the case of almost all engineering problems this experiment does not have a unique answer. The restrictions for the system are chosen in a way for the problem not to have a straight-forward answer. The students have to compromise between the design requirements.

3. The experiment also serves as a practical control problem for which the previously learned control background can be used to design the control gains. The shortcoming of the linear controllers is a practical introduction for the student to think about nonlinear control solutions.

4. The SMA robotic experiment also provides the means for investigating more advanced properties of Shape Memory Alloy like the interdependency of the transformation temperatures and material stress. This property makes the use of the SMA wire as a position sensor more challenging.

5. The lectures, lab sessions, reports, and presentations educate students on solving real-life engineering problems and help them to be prepared for their job as engineers. 
6. The experiment has a level of challenge for the students who are interested to in more details to this problem. Taking this course has inspired several students to use smart materials in their senior design projects.

7. Analyzing the course evaluation data shows that the majority of the students have found this experiment interesting and they think that they had a better learning experience. This is in part because most of the students have had no experience with shape memory alloys before. Another contributing factor is the way that the experiment was conducted; the students had to design their own lab procedures. Also the communication of the results in the form of reports and presentations played a major role in making the lab more attractive and a better learning experience.

\section{References}

[1] Moini, Hossein, "Active Materials \& Microcontroller Applications in Design of Intelligent Systems," 1998 Annual Conference (Seattle: ASEE, June1998).

[2] Penrod, Luke, Talley, Diana, Froyd, Jeff, Caso, Rita, Lagoudas, Dimitris, and Kohutek, Terry, "Integrating smart materials into a first-year engineering curriculum: A case study," Proceedings - Frontiers in Education Conference, v 2 200: F3B/21-F3B/26 (IEEE cat n 02CH37351).

[3] Alley, Michael, and Harry Robertshaw, "Interweaving Engineering and Writing: A Model Large-Laboratory Course at Virginia Tech," 2001 ASEE Annual Conference (Albuquerque: ASEE, June 2001).

[4] Alley, Michael, and Harry Robertshaw, "An Effective Incorporation of Student Presentations Into a Large Laboratory Course," 2001 ASEE Annual Conference (Albuquerque: ASEE, June 2001).

[5] Elahinia, Mohammad, and Hashem Ashrafiuon, "Nonlinear control of a shape memory alloy actuated manipulator," Journal of Vibration \& Acoustics - Transactions of the ASME, October 2002, 124(4): 566-575.

Mohammad H. Elahinia (M.S., Villanova University, 2001) is a Ph.D. candidate in the ME Department of Virginia Tech; he is presently associated with the Advanced Vehicle Dynamics Laboratory. His research interests are in developing control strategies for smart structures. Having taught ME lab and classes for more than 2 years, he has developed a new problem solving approach to teach smart materials to Mechanical Engineering students. 


\section{Appendix A - Memorandum}

\section{MicroRobotics, Inc. Blacksburg, VA 24060}

August 27, 2003

To: $\quad$ Pat Philips, Engineer

From: Kelly Smith, Project Manager

Subject: Request for Testing of a Shape Memory Alloy Manipulator

As discussed in our previous meeting, our company has been asked to develop a manipulator for a miniature mobile robot $(10 \mathrm{~cm}$ by $10 \mathrm{~cm}$ by $10 \mathrm{~cm})$. Needing to operate in the desert, the robot should be capable of sensor deployment, adjustment, and replacement. For that reason, the manipulator must be lightweight and relatively powerful to pick up and work with objects. Our manufacturing group has designed and built a one-degree-of-freedom manipulator that is actuated by the Shape Memory Alloy (SMA) wire. SMA was chosen because of its light weight, sensing capabilities, and compatibility with the environment. This memo requests you to find an appropriate control system for the manipulator.

Because the mobile platform is still under development, we have installed the manipulator on a stationary base. You can use this model for testing (for a cutaway drawing of the prototype, see Figure 2). The manipulator is initially positioned at approximately $\theta=-45^{\circ}$, and the SMA wire is pre-stressed by the linear spring. The wire is heated by the Joule heating. When the temperature exceeds the activation start temperature, the wire starts the phase transformation. As a result of this transformation, the wire contracts, thus applying torque to the moving arm. Therefore, the arm can be positioned anywhere between $-45^{\circ}$ and $+90^{\circ}$. The hanging mass simulates the gripper and the payload.

Your job is to test the model and choose the most appropriate set of controller gains. The manipulator's task is to pick up sensors from the platform (lower position) and to put them on an object (horizontal position \pm 1 degree) in less than 1 second. The manipulator is then to remain horizontal before it picks up the next sensor. On a typical mission, the robot needs to deploy 60 sensors per hour. The mobile robot is autonomous; therefore, the manipulator's is internally powered by an alkaline 9-volt battery. Each battery has a capacity of 350 milliamp-hours (mAh). Required is that the manipulator can work for 1 hour using this 9-volt battery. Your choice of the control gains should satisfy both the task requirement and the power restriction.

After designing the controller, please test it for three other desired angular positions (set-points). Comment on the controller's ability to reach those set-points. Also, evaluate your controller's ability to follow a joystick. Document your design procedure and justify your 
final choice for control gains. Will the proposed controller work? If you are suggesting a different control design, please document and justify your design.

Our manufacturing group has initially installed an encoder for sensing the angular position of the manipulator. Because the robot has to perform in hostile environments, you are required to reduce the number of sensitive items such as the encoder. It has been suggested that the electrical resistance, a physical property of SMA that characteristically changes with phase transformation, can be used to determine the state or phase of the SMA actuators and, therefore, can be used for closed loop control. Please determine if it is, in fact, possible to use the measured resistance of the wire to determine the angular position of the manipulator. Please report your results.

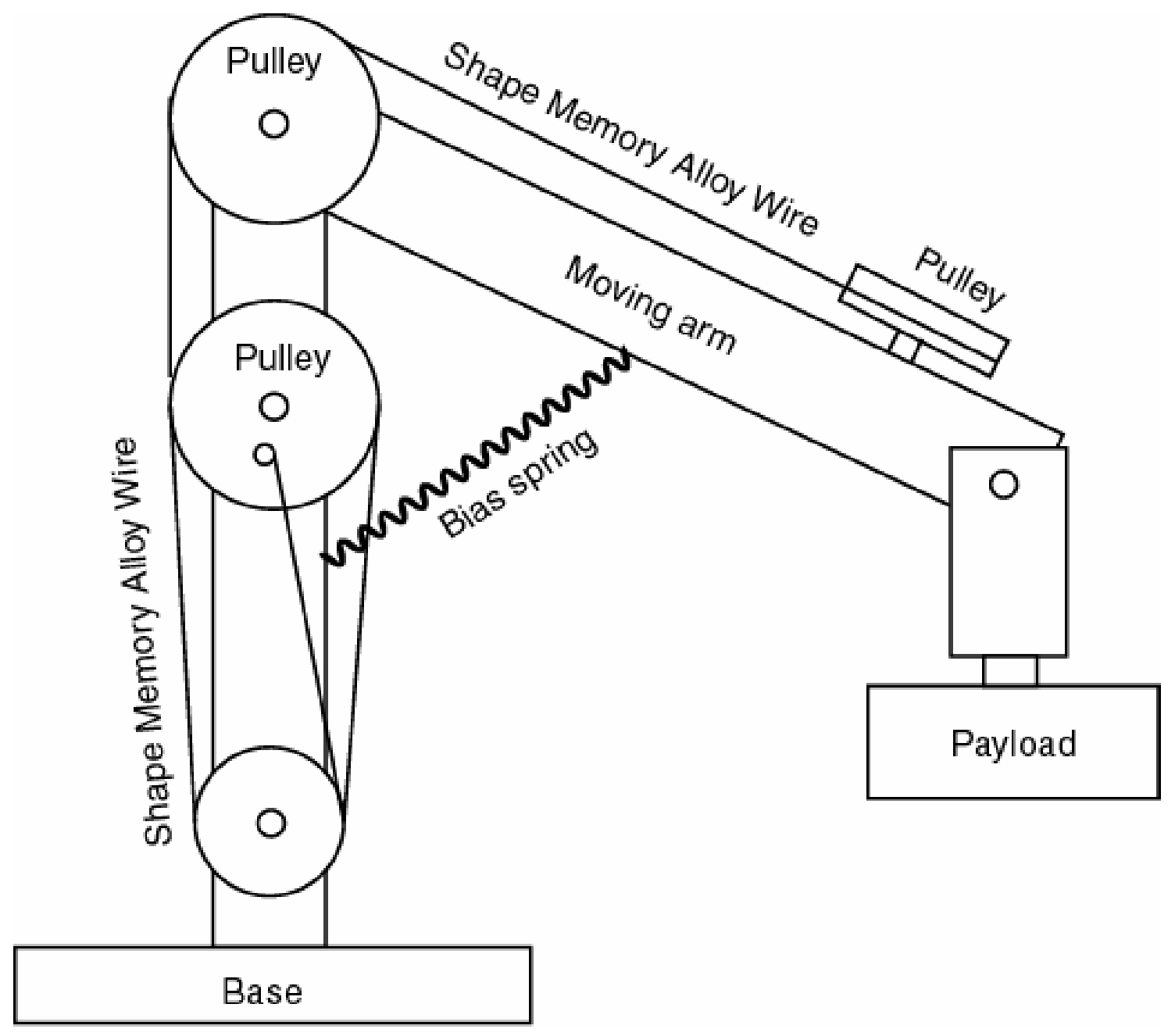

Figure 2. Shape Memory Alloy actuated manipulator to be installed on the mobile platform. 